Perceived social norms of health behaviours and college engagement in British students

Dr John McAlaney, PhD*

Psychology Research Group

Bournemouth University

Bournemouth, BH4 9HW

United Kingdom

Email jmcalaney@bournemouth.ac.uk

William Jenkins

Community Engagement Manager

Tech Stories

* Corresponding author 


\section{Perceived social norms of health behaviours and college engagement in British students}

Abstract

Aims

The social norms approach is an increasingly widely used strategy of behaviour and attitude change that is based on challenging misperceptions individuals hold about their peers. Research to date has been carried out predominately in the American college system, with a focus on substance use behaviours. The aim of the current study was to explore peer perceptions of both substance use and other behaviours in a British student sample, as the first step of determining whether the social norms approach may be applicable within Europe.

Methods

Students at eight Further Education colleges in the UK were surveyed on their personal and perceived peer health and college engagement behaviours and attitudes by means of a printed and online survey.

Results

Respondents reported a perceived norm of frequency of substance use that was higher than the reported norm. Results relating to the injunctive norms of substance use were mixed but demonstrated that the majority of respondents do not actively approve of tobacco, cannabis or other drug use. Respondents also reported a norm of academic engagement that was more positive than the perceived norm of their peers.

Conclusions

The results around substance use are consistent with work conducted in the American college system, despite the differences in culture and legislation. In addition there results indicate that there 
may be similar misperceptions around other areas of health and college engagement. This suggests that the social norms approach may be a viable method of behaviour change in UK students.

Key words: Social norms, college students, health, educational attainment 
Introduction

Whilst national surveys suggest that rates of alcohol consumption and tobacco smoking are decreasing overall in the UK young adults remain the highest risk group (Office for National Statistics 2014). Late adolescence often coincides with entry into higher or further education, which is associated with increased substance use (Schulenberg and Maggs 2002; Akmatov et al. 2011), with students reported to have a higher level of alcohol consumption than non-students (Kypri et al. 2005; Webb et al. 1998). Excessive alcohol use in young adults has been found to be associated with cognitive and neurological impairments (Hartley et al. 2004; Monti et al. 2005). Within student populations substance use has also been found to be predictive of unsafe sexual practices, physical and psychological harms as well as delays in academic progression (Bergen et al. 2005; Boot et al. 2007; Ham and Hope 2003; Akmatov et al. 2011). In addition it has been reported that $23 \%$ of $16-$ 24 year olds in the UK are cigarette smokers (Office for National Statistics 2014). Smoking initiation in adolescence is associated with a trajectory of smoking throughout adulthood (Sweeting and West 2001). As such adolescence and early adulthood represent a crucial period in which to target and alter behaviours and attitudes. Educational settings provide a unique opportunity to engage with these populations.

Work originating in the American college system (Perkins and Berkowitz 1986; Turner et al. 2008) has demonstrated that students tend to overestimate both how frequently and heavily (the descriptive norm) their peers use psychoactive substances, and how socially acceptable they believe these behaviours to be (the injunctive norm). This finding has become the basis of a form of intervention and prevention known as the social norms approach. It operates on the premise that these misperceptions act as a form of social influence on the individual to match what they believe to be the group norm, a process predicted by social psychological theories (Festinger 1954; Borsari and Carey 2003). In the case of substance use a student may for example erroneously perceive their university or college peers to be heavier and more frequent consumers of alcohol than is actually the 
case. By challenging these misperceptions through a variety of means such as mass media campaigns and personalised online feedback the substance use of the individual may be reduced. The approach has become widely used in the USA and appears to be a viable method of prevention and behaviour change (McAlaney et al. 2011; Moreira et al. 2009). Examples include work at the University of Virginia (Turner et al. 2008), which found that the risks of students reporting multiple, negative alcohol related consequences fell by over half following the implementation of a social norms campaign. Similarly the 'Most of Us' marketing campaign originating in Montana in the USA has been used to address behaviours such as cigarette smoking in adolescents (Linkenbach and Perkins 2003).

To date the majority of social norms studies have taken place on American college campuses, with a focus on substance use behaviours. The work which has been done in the UK and Europe suggests that similar misperceptions of substance are evident in European student populations (McAlaney and McMahon 2007; McAlaney et al. 2012; Page et al. 2008), however there remains a need to further determine the nature of these misperceptions within UK students, particularly as the social norms approach becomes more popular in Europe (McAlaney et al. 2011). It cannot be assumed that research developed in the American college system is applicable to European settings, given the differences in legislation, culture and educational systems (Wicki et al. 2010). In addition the majority of published research studies in the USA and the UK have looked at university settings. There has been little work in colleges in the UK. These institutions are part of further education (FE) rather than higher education (HE) and often include students aged between 16 to 18 , with an emphasis on vocational courses. Work in Finland has suggested that misperceptions of substance may be particularly prevalent and influential in younger adolescent populations (Lintonen and Konu 2004). Therefore there is a need to extend research on social norms in educational settings such as FE colleges where the student population is likely to consist of younger people than previous research has been based on. 
Furthermore in comparison to substance use there is substantially less social norms research on other aspects of student health, well-being and academic achievement. It is important that these other areas are explored, as it may be that the social norms approach could be used to bring about behaviour and culture change in the same way that it has for substance use. Exploration of the possibilities of the social norms is of particular importance in student populations, where it could be argued social norms could be argued to be particularly influential. For example research with American college students has demonstrated that alcohol consumption in student populations is often associated with socialisation and group activities (Wicki et al. 2010). Students attending the same college or university may also be in close contact with one another, further heightening the influence of social norms in this group (Borsari and Carey 2003).

However whilst it is acknowledged that perceptions of the behaviours and attitudes of other students overall is important recent research has begun to examine in more detail the role of reference group and norm salience. It has been found for instance that gender specific social norms interventions are more effective among female students with students who identify strongly with their gender (Lewis and Neighbors 2007). The perceived norm has also been found to be more strongly predictive of personal behaviour in American college student population when the reference group used matches the individual in terms of gender, ethnicity and Greek affiliation status (Neighbors et al. 2010). There is a lack of research which explores how students at FE colleges in the UK socialise with each other and how salient they see the perceived norms of their college to be to them.

The current study reports results from the University and College Social Norms Survey (UCSNS), which aimed to address these gaps in the literature by investigating social norms in UK students on a wider range of behaviours, with the inclusion of measures of perceived typicality and time spent socialising with other students. A total of 163 colleges and universities participated in the survey. In order to secure the participation of the institutions involved a collaborative approach was taken, in 
which the topics covered by the survey and the question format was the result of negotiations between the researchers and the stakeholders from the institutions. As the main aim of the overall project was to explore the feasibility of implementing social norms campaigns a quantitative approach was taken, as demonstrating a numerical difference between personal and perceived behaviours/ attitudes is a prerequisite of such campaigns.

Due to wide range of sample sizes that were obtained from each institution this paper is limited to the eight FE colleges that returned the highest number of respondents, as outlined below. 
Methodology

The eight participating colleges were located with England, spread between the North, the Midlands and the South East. Each college is based in a primarily urban area of overall similar levels of socioeconomic deprivation. Data was collected from students at each institution using a combination of printed and online surveys. Printed surveys were disseminated in compulsory module classroom sessions by college tutors. The selection of which classroom groups to invite to participate in the research was driven by the logistical factors inherent in a large multi-site study reliant on third parties, namely staff time and availability. Due to the large scale of the project and the reliance on the assistance of third parties to collect the data it was not possible to accurately record how many students in each classroom session agreed to participate. However feedback from college tutors indicated that very few students declined participation in the survey.

An online version of the survey was made available at each institution, with students invited by college tutors to complete the surveys whilst in a computer lab as part of a timetabled session. However the link was available for use outside of timetabled sessions for any student who wished to complete the survey in their own time. Participation in the survey was incentivised through use of a prize draw which offered students the opportunity to win a range of prizes including a laptop or a mountain bike. Both printed and online surveys were tailored to include the logo and name of the college.

Respondents were asked to report their personal behaviour or attitude and their perceived behaviour or attitude of their same age and same gender peers at their institution. The survey items covered frequency of alcohol, tobacco, cannabis and other drug use; importance of obtaining good grades; participation in clubs; volunteering activities; as well as personal safety behaviours of riding in a car with a drunk driver and leaving both drinks and belonging unattended in public places. Items querying frequency of a behaviour used response options on a 12 point scale from 'Never' to 'Every day or nearly every day'. The personal behaviour item on smoking asked respondents if they had 
smoked a cigarette in the last week, with a response option of yes or no. The corresponding perceived norm item asked participants what percentage of their same age and sex peers they thought would have smoked a cigarette in the last week. Response options to this and other items which used a yes/ no format for the personal behaviour question consisted of ' $0 \%, 1-9 \%, 10-$ $19 \% . .$. ' and so on up until ' $90-99 \%, 100 \%$ '.

As has been debated extensively in the research literature there are a number of different ways of measuring alcohol consumption, depending on whether beverage specific items and measure size are included (Greenfield 2000). As measures of alcohol consumption become more precise the language they use also tends to become more technical and extensive. As such a balance must be reached between obtaining the level of precision sought by the researcher and the realities of what type of question format will be accessible for the target population. After discussions with the colleges and other stakeholders it was decided that the most appropriate approach would be to simply ask participants about the number of alcoholic drinks they consume. This provided a less precise measure of alcohol consumption that could have otherwise been achieved, however it is important to note that the primary focus of the study was to determine if there was a discrepancy between personal and perceived behaviour, rather than to explore personal behaviour in-depth. More detailed data on alcohol consumption in young adults is already available through a number of national studies, such as the Opinions and Lifestyle Survey (Office for National Statistics 2014).

The number of alcoholic drinks consumed on a night out (analogous to the phrase 'partying' in American college settings) was measured as a continuous number, capped at 15 . Number of sexual partners in the last year was recorded using the same format. Injunctive norms of attitudes towards tobacco, cannabis and other drug use were measured on a five point scale from 'strongly disapprove' to 'strongly approve'. The degree to which respondents socialise with other students was measured on a five point scale of 'Never', 'Rarely', 'Sometimes', 'Often' and 'Very often'. How 
typical a student of their institution that they perceived themselves to be was rated on a five point scale from 'Very untypical' to 'Very typical'.

Ethical approval for the study was given at the time of it by the ethical committee of the institution of the first author. It was noted that the survey could involve participants, particularly those aged under 18 , reporting what may be illegal behaviours. However in keeping with previous research into these age groups it was felt that ensuring survey data was secure and anonymous would be sufficient to negate any harmful consequences to the participants. As commented the study was developed with through extensive discussion with the participating colleges, who were aware of these issues and had their own policies on how undertake work in these types of behaviours . Students were advised both on the survey forms and by the college tutors that participation in the survey was entirely voluntary and that their responses to the survey would be anonymised. The paper surveys included a cover page where the student was asked to enter their contact details if they wished to be entered into the prize draw. This cover sheet was then removed from each survey before data entry began, thus separating any personally identifying information from the personal behaviour items. Data from the online surveys was downloaded from a secure server and then split into two files, again consisting of one file with personal contact details for the purposes of the prize draw and one file of the remaining survey data. After the prize draw had been conducted the personal contact information of all students was destroyed.

Data analysis

In order to demonstrate the gap between the reported norms and perceived norms the institution with the highest number of respondents $(n=722)$ was selected and use to create table 1 . This depicts the reported norm for each behaviour/ attitude and the percentage of students at the institution who perceived the norm to be lesser than, equal to or greater than the reported norm. 
Although not shown here a similar analysis was conducted for each of the other 7 institutions individually. There were no substantial differences found between the responses of these intuitions and the data presented in table 1.

The data from all 8 institutions was then used for inferential data analysis. Multiple regression and binary logistic regression was used to determine the predictors of personal behaviours and attitudes, depending on whether the outcome measure was recorded as a dichotomous categorical variable or a continuous variable. Within each regression model the corresponding perceived social norms for the behaviour or attitude was included a predictor of the outcome behaviour or attitude. For example, the perceived alcohol consumption frequency of peers (i.e. the perceived social norm) was included as a predictor of personal frequency of alcohol consumption. All variables were entered simultaneously into the models to evaluate unique contributions (Cohen et al. 2003). 
Results

Completed surveys were returned by 4,048 respondents, of which $50 \%$ were male and $50 \%$ were female. In terms of survey format $56 \%$ of respondents completed the online survey and $43 \%$ completed a paper survey. Respondents had a mean age of $18.2(S D=3.6)$, with $52 \%$ of respondents being aged under 18 and $48 \%$ being aged 18 or over. Comparisons between personal behaviour or attitude and perceived peer behaviour or attitude are shown in table 1 . The reported norm of each behaviour or attitude was calculated as the median value for those items measured on a continuous scale and as the majority response for the items with dichotomous yes/ no response option.

$32 \%$ of students reported that they perceived themselves to be untypical or very untypical of the student population of their institution. A small number reported that they never $(5 \%)$ or rarely $(10 \%)$ socialised with other students, with the remainder reporting that they did so sometimes (25\%), often (34\%) or very often (27\%).

The results of the multiple regression analysis are shown in tables 2 to 7 . Multicollinearity diagnostics were conducted on each regression model. None of the tolerance factors were lower than 0.91 and none of the variance inflation factors (VIF) were greater than 1.099, indicating that there were no serious issues with multicollinearity. All of the regression models were significant ( $p$ $<.001)$. The perceived social norm was a significant predictor in all of the models. 
Discussion

The results of the study suggest that respondents at the selected institution perceive the norm of frequency of alcohol, cannabis and other drug use to be higher than the reported norm. The gap between the perceived norm and the reported norm was especially notable with regards to having smoked a cigarette in the last week, where $96 \%$ of respondents stated that they perceived the norm to be higher than the reported norm. Similar patterns were evident with the sexual health behaviours of number of sexual partners in the last year and frequency of unsafe sex, with a high percentage of respondents stating a perceived norm that was higher than the reported norm. Approximately two-thirds of participants perceived the norm of willingness to be a passenger in a car driven by a drunk driver to higher than the reported norm. However a smaller percentage of respondents stated that their perceived norm of leaving drinks and belonging unattended in public places was higher than the reported norm.

There were a number of statistically significant $(p<.05)$ gender effects. Male respondents were found to drink more alcohol and to be more likely to smoke cannabis and use other drugs more frequently than female respondents. There was no significant predictive effect of gender on frequency of alcohol use or tobacco use; although it was noted that male respondents were significantly more likely to approve of both tobacco use and cannabis use. Female respondents were more likely to report that obtaining good grades was important to them. Female respondents were also more likely to make use of college support services, but less likely to participate in clubs and societies. In terms of sexual health male respondents were found to be more likely to report having engaged in unsafe sex than female students. The gender effects found across all behaviours could be characterised as male respondents being more likely to participate in risky behaviours, although it should also be commented that whilst statistically significant many of the gender effects that were found are relatively weak. Overall age was not a significant predictor of either behaviour or attitudes, which may be a reflection of the narrow age range evident in the sample. 
It is encouraging that the norm of importance of good grades was high amongst the sample, although the majority of respondents stated that their perceived norm was lower than the reported norm. In addition to the noted work by Rosenthal and Jacobson (1966) more recent research into teacher expectations and classroom context have demonstrated the role of social influence factors in academic achievement (Straehler-Pohl et al. 2014). Approximately half ( $54 \%$ and $43 \%$ of respondents respectively) of respondents stated that their perceived norm of being involved in clubs and societies and making use of support services was lower than the reported norm. This is of relevance as engagement with the educational experience has been to found to be a predictor of academic performance (Lee 2014), and the type of skills gained through participation in clubs and skills are relevant to the employability, a topic which is of increasing relevance to university and college students (Maurer and Mawdsley 2014).

These results would appear to support previous work conducted primarily in the USA which has suggested that students overestimate rates of risky or negative behaviour and attitudes in their peers. As demonstrated by the regression models the perceived norm was a significant predictor of every behaviour and attitude which was included as an outcome measure. This provides support to the view that the social norms approach could be used to reduce rates of harmful behaviours and attitudes in UK college student populations, in the same way that it has been successfully applied in American educational systems. The results of the current study also suggest that the social norms approach may also be of use in behaviours out with substance use and health, where the bulk of previous research has been focussed. For instance if it is the case that students are indeed underestimating the importance that their peers place on good grades then this misperception could be challenged through use of a social norms campaign, which would promote the positive message that the majority of students at an institution place high importance on academic achievement. This may help counter the trend noted by Galvan et al. (2011) in which more negative academic behaviours start to become valued as pupils move from primary to secondary education. 
Challenging misperceptions on a wide range of topics may have additional benefits beyond changes to the targeted behaviours and attitudes. Research in North America has found that students reported that they felt happier studying at their institution following the application of a social norms campaign, which may be because the campaign helped the students to realise that they were not in fact atypical from their peers for not engaging in frequent and heavy alcohol consumption (Perkins 2007). In addition it has been noted that the sense of belonging a student has at an educational institution can be linked to their academic achievements (Van Houtte and Van Maele 2012). A more holistic social norms campaign which targets a number of different aspects of college life could be used to help develop this sense of belonging.

The results of the injunctive norms of the substance use items were mixed. The majority of respondents (75\%) stated a perceived norm of tobacco use attitude which matched the reported norm, specifically that tobacco use would be neither approved nor disapproved. With regards to cannabis use however nearly half $(48 \%)$ of respondents stated that they perceived disapproval of cannabis to be higher than the reported norm. This is in contrast to the social norms literature, in which studies have tended to find that people perceive the behaviour or attitude of their peers to be more negative than the reported norm would suggest is the case. As observed by LaBrie et al. (2011) there is a lack of research on the injunctive norms of cannabis use, who also found complex relationships between personal cannabis use, personal approval and the perceived approval of different reference groups. This highlights the need for more in-depth research into injunctive norms of substance use, particularly with regards to substances such as cannabis which have differing legal statuses between countries. In the UK the possession of cannabis is illegal, although police officers can issue a warning for possession of small amounts. The classification of cannabis in the UK has also changed several times in recent years. Legislation may serve as an indicator to individuals as to what the social norms and values of society are, and therefore the legal ambiguity of cannabis use may in part explain the inconsistencies found with injunctive norms of cannabis in the current study. The results regarding approval of other drug use was more consistent with previous social norms 
research, with the majority of respondents stating perceived norm of disapproval that was lower than the reported norm.

There is relatively little research on the role of injunctive norms compared to research on descriptive norms (McAlaney et al. 2011), but it has been argued that the latter may be more influential (LaBrie et al. 2010). This may be because they are seen by the individual to represent the beliefs of the group to which they belong, whereas descriptive norms are more prone to situational effects such as availability of the substance (Jacobson et al. 2011). As noted elsewhere injunctive norms can be used as the basis for a social norms campaign when the descriptive norm is unhealthy or unsuitable (Mollen et al. 2013). Despite the fact that in the current study the vast majority of students reported never using drugs it may be that parents and stakeholders would object to a campaign which acknowledged that even a small amount of drug using was taking place, particularly given that many of the students are minors. As such a social norms campaign which is instead based on a message that students do not approve of drug use may be more acceptable. If it is the case though that injunctive norms are more deeply imbedded then this may in turn also make them more resistant to be being challenged. It has been observed for instance that social norms based interventions for cannabis use may be less successful and changing perceived injunctive norms than they are descriptive norms (Elliott et al. 2014).

Time spent socialising with other students was a significant predictor of alcohol and tobacco use, but not of cannabis and other drug use. This may be a reflection of the fact that cannabis and other drug use were reported to be uncommon. Similarly perceived typicality of a student of the referent institution was a significant predictor in several models, but not all of them. It is of interest to note that the behaviours where perceived typicality was a significant predictor (e.g. alcohol use and cannabis use) are those which could be argued to be behaviours which are associated with the stereotype of a student. This may suggest the influence of a wider perceived social norm of students in general, which transcends the perceived norms of the student's own institution. This does 
contrast somewhat with previous suggestions the referent group of other individuals at the students' university or college may be quite a distal and undefined group to the individual (LaBrie et al. 2010), and therefore one which does not exert much social influence. An even further removed and more abstract group such as students in general could therefore be expected to be even less important in determining individual behaviour. However as noted by McShane and Cunningham (2003), with regards to Canadian students, portrayals of the stereotypical student in American media and TV shows appeared to be more influential on Canadian students than the norms of students from their own country. This research and the results from the current study highlight the need to better understand how students engage with norm messages and which reference groups are the most relevant and salient. This in turn would allow for more targeted and effective social norms campaigns.

When considering the results of the current study a wider issue in the social norms field must be acknowledged. This is the use of phrases such as 'misperceptions' when describing discrepancies between reported norms and perceived norms, which has been the subject of debate in the literature (Pape 2012; Perkins 2012). To be able to confidently identify a perception as a misperception it would be necessary to demonstrate that the reported norm is indeed the actual norm, and that the sample is representative of the student population. Addressing this debate goes beyond the scope of the current paper but it is acknowledged that there may be issues with the assumption that any apparent overestimations or misperceptions are genuine. As such in the current paper we have avoided identifying the discrepancies between the reported and perceived norms as definite misperceptions.

The study relied on self-report, which may undermine the validity of results. From a research perspective the use of a mixture of different types of response options was not ideal, as this necessitates different analysis strategies and it turn makes it more difficult to make comparisons between behaviours and attitudes. As stated previously this arose from the fact that the content and 
phrasing of the survey was a compromise between the researchers and the 163 individual institutions involved. This negotiation of content was based primarily around the areas that would be covered in the survey, and resulted in a survey that was longer than would have otherwise been used by the researchers. This could have led to a degree of response acquiescence, in which respondents stop fully reading questions and instead just tick the same response options repeatedly for each item. The study was also cross-sectional, which negates any conclusions around whether perceptions are indeed a causal factor of behaviour and attitudes. However previous research in the social norms field has suggested that perceptions are the cause of behaviours, albeit with a degree of reciprocal causality (Neighbors et al. 2006). Finally the use of online surveying combined with paper surveying could have resulted in students completing the survey more than once. Given the reliance on individual colleges to administer the surveys it is difficult to comment on how much of a risk this issue was. It was observed though that the majority of online surveys were completed within the scheduled timetabled slot, rather than at a later time. In order to dissuade respondents from completing the survey multiple times to increase their change of winning the prize draw college tutors were asked to advise students that each respondent would only be eligible for one ticket in the prize draw. Analysis of the personal contact information provided by respondents so that they could participate in the prize draw found only four instances in which a respondent appeared to have completed the survey twice.

In keeping with the ethos of the social norms approach it must finally be observed that the majority of students in the current study reported substance use and behaviours and attitudes which were healthier and more positive than perhaps could be expected from the stereotype of students. This reflects research into alcohol use in the UK which suggests that, despite being the highest risk group, the majority of young adults do not drink alcohol frequently and heavily (Office for National Statistics 2010). It has been argued that the negative and at times alarmist rhetoric applied to substance use and other health behaviours in young adults may itself be a contributing factor towards the creation and maintenance of substance use misperceptions (Perkins 2003). This is not to 
say that substance use in this group should be ignored; as previously discussed substance use in young adults can be associated with numerous negative consequences. However it does highlight the importance of discussing the issues with an appreciation of the wider context of actual substance use rates. This fits with UK government policy (Ellison 2013), which states that in order to reduce harmful drinking behaviour should be changed so that heavy drinking is not seen as being acceptable. This is precisely what the social norms approach aims to achieve, through demonstrating to people that their peers are not as accepting of negative behaviours and attitudes as they may assume. In light of the criticisms of the criticisms of more traditional forms of substance misuse education (Foxcroft et al. 2003) the current study provides support to exploring new avenues of prevention and behaviour change such as the social norms approach.

Declaration of Interest

The second author was previously employed by a private company which assisted in the data collection. 
References

Akmatov, M. K., Mikolajczyk, R. T., Meier, S., \& Kramer, A. (2011). Alcohol consumption among university students in north rhine-westphalia, germany-results from a multicenter crosssectional study. Journal of American College Health, 59(7), 620-626, doi:10.1080/07448481.2010.520176.

Bergen, H. A., Martin, G., Roeger, L., \& Allison, S. (2005). Perceived academic performance and alcohol, tobacco and marijuana use: Longitudinal relationships in young community adolescents. Addictive Behaviors, 30(8), 1563-1573, doi:10.1016/j.addbeh.2005.02.012.

Boot, C. R. L., Vonk, P., \& Meijman, F. J. (2007). Health-related profiles of study delay in university students in The Netherlands. International journal of adolescent medicine and health, 19(4), 413-423.

Borsari, B., \& Carey, K. B. (2003). Descriptive and injunctive norms in college drinking: A metaanalytic integration. [Article]. Journal of Studies on Alcohol, 64(3), 331-341.

Cohen, J., Cohen, P., West, S. G., \& Aiken, L. S. (2003). Applied Multiple Regression/Correlation Analysis for the Behavioral Sciences Mahwah, NJ: Lawrence Erlbaum.

Elliott, J. C., Carey, K. B., \& Vanable, P. A. (2014). A preliminary evaluation of a web-based intervention for college marijuana use. [Article]. Psychology of Addictive Behaviors, 28(1), 288-293, doi:10.1037/a0034995.

Ellison, J. (2013). Reducing harmful drinking. In D. o. Health (Ed.).

Festinger, L. (1954). A theory of social comparison processes. Human Communications, 7, 117 - 140.

Foxcroft, D., Ireland, D., Lister-Sharp, D. J., \& Breen, R. (2003). Longer-term primary prevention for alcohol misuse in young people: A systematic review. Addiction, 98, 397 - 411.

Galvan, A., Spatzier, A., \& Juvonen, J. (2011). Perceived norms and social values to capture school culture in elementary and middle school. [Article]. Journal of Applied Developmental Psychology, 32(6), 346-353, doi:10.1016/j.appdev.2011.08.005.

Greenfield, T. K. (2000). Ways of measuring drinking patterns and the differences they make: experience with graduated frequencies. Journal of Substance Abuse, 12, 33 - 49.

Ham, L. S., \& Hope, D. A. (2003). College students and problematic drinking: a review of the literature Clinical Psychology Review, 23(5), 719 - 759.

Hartley, D. E., Elsabagh, S., \& File, S. E. (2004). Binge drinking and sex: Effects on mood and cognitive function in healthy young volunteers. Pharmacology, Biochemistry and Behavior, 78(611 619).

Jacobson, R. P., Mortensen, C. R., \& Cialdini, R. B. (2011). Bodies obliged and unbound: differentiated response tendencies for injunctive and descriptive social norms. Journal of Personality and Social Psychology, 100(3), 433-448, doi:10.1037/a0021470.

Kypri, K., Cronin, M., \& Wright, C. S. (2005). Do university students drink more hazardously than their non-student peers? Addiction, 100(5), 713-714, doi:ADD1116 [pii]

10.1111/j.1360-0443.2005.01116.x.

LaBrie, J. W., Hummer, J. F., \& Lac, A. (2011). Comparing injunctive marijuana use norms of salient reference groups among college student marijuana users and nonusers. Addictive Behaviors, 36(7), 717-720, doi:10.1016/j.addbeh.2011.02.004.

LaBrie, J. W., Hummer, J. F., Neighbors, C., \& Larimer, M. E. (2010). Whose opinion matters? The relationship between injunctive norms and alcohol consequences in college students. Addictive Behaviors, 35(4), 343-349, doi:10.1016/j.addbeh.2009.12.003.

Lee, J. S. (2014). The relationship between student engagement and academic performance: Is it a myth or reality? [Article]. Journal of Educational Research, 107(3), 177-185, doi:10.1080/00220671.2013.807491.

Lewis, M. A., \& Neighbors, C. (2007). Optimizing personalized normative feedback: The use of gender-specific referents Journal of Studies on Alcohol and Drugs, 68, 228 - 237. 
Linkenbach, J., \& Perkins, H. W. (2003). Most of us are tobacco free: an eight month social norms campaign reducing youth initiation of smoking in Montana. In H. W. Perkins (Ed.), The Social Norms Approach to Preventing School and College Age Substance Abuse: A Guide for Educators, Counselors, Clinicians. San Francisco: Jossey-Bass.

Lintonen, T., \& Konu, A. (2004). The misperceived social norm of drunkenness among early adolescents in Finland. Health Education Research, 19(1), 64 - 70.

Maurer, H., \& Mawdsley, J. (2014). students' skills, employability and the teaching of european studies: challenges and opportunities. [Article]. European Political Science, 13(1), 32-42, doi:10.1057/eps.2013.34.

McAlaney, J., Bewick, B., \& Hughes, C. (2011). The international development of the 'Social Norms' approach to drug education and prevention. Drugs: Education, Prevention, and Policy, 18(2), 81-89, doi:10.3109/09687631003610977.

McAlaney, J., Boot, C. R., Dahlin, M., Lintonen, T., Stock, C., Rasmussen, S., et al. (2012). A comparison of substance use behaviours and normative beliefs in North West European university and college students. International Journal of Disability and Human Development, 11(3), 281 - 287.

McAlaney, J., \& McMahon, J. (2007). Normative beliefs, misperceptions and heavy episodic drinking in a British student sample. Journal of Studies on Alcohol and Drugs, 68(3), 385 - 392.

McShane, K. E., \& Cunningham, J. A. (2003). The role of relevancy in normative feedback for university student's drinking patterns. Addictive Behaviours, 61, 794 - 798.

Mollen, S., Rimal, R. N., Ruiter, R. A., Jang, S. A., \& Kok, G. (2013). Intervening or interfering? The influence of injunctive and descriptive norms on intervention behaviours in alcohol consumption contexts. Psychol Health, 28(5), 561-578, doi:10.1080/08870446.2012.752827.

Monti, P. M., Miranda, R., Nixon, K., Sher, K. J., Swartzwelder, S., Tapert, S. F., et al. (2005). Adolescence: Booze, brains and behaviour. Alcoholism: Clinical and Experimental Research, 29(2), 207 - 220.

Moreira, M. T., Smith, L. A., \& Foxcroft, D. (2009). Social norms interventions to reduce alcohol misuse in University or College students. (Vol. The Cochrane Library 2009 Issue 3): The Cochrane Collaboration.

Neighbors, C., Dillard, A. J., Lewis, M. A., Bergstrom, R. L., \& Neil, T. A. (2006). Normative misperceptions and temporal precedence of perceived norms and drinking. Journal of Studies on Alcohol, 67, 290 - 299.

Neighbors, C., LaBrie, J. W., Hummer, J. F., Lewis, M. A., Lee, C. M., Desai, S., et al. (2010). Group Identification as a Moderator of the Relationship Between Perceived Social Norms and Alcohol Consumption. [Article]. Psychology of Addictive Behaviors, 24(3), 522-528, doi:10.1037/a0019944.

Office for National Statistics (2010). General Lifestyle Survey 2008: Smoking and Drinking among Adults. London: Office for National Statistics.

Office for National Statistics (2014). Opinions and Lifestyle Survey.

Page, R. M., Ihasz, F., Hantiu, I., Simonek, J., \& Klarova, R. (2008). Social normative perceptions of alcohol use and episodic heavy drinking among Central and Eastern European adolescents. Substance Use and Misuse, 43, 361 - 373.

Pape, H. (2012). Young people's overestimation of peer substance use: an exaggerated phenomenon? Addiction, 107(5), 878-884, doi:10.1111/j.1360-0443.2011.03680.x.

Perkins, H. W. (2003). The emergence and evolution of the social norms approach to substance abuse prevention. In H. W. Perkins (Ed.), The social norms approach to preventing school and college age substance abuse: A handbook for educators, counselors and clinicians (pp. 3 17): Jossey-Bass.

Perkins, H. W. (2007). Misperceptions of Peer Drinking Norms in Canada: Another Look at the 'Reign of Error' and Its Consequences among College Students. Addictive Behaviors, 32, 2645 2656. 
Perkins, H. W. (2012). Misperceptions of peer substance use among youth are real. Addiction, 107(5), 888-889, doi:10.1111/j.1360-0443.2012.03782.x.

Perkins, H. W., \& Berkowitz, A. D. (1986). Perceiving the community norms of alcohol use among students: Some research implications for campus alcohol education programming. International Journal of the Addictions, 21, 971 - 976.

Rosenthal. R, \& Jacobson, L. (1966). Teachers expectancies - determinants of pupils' iq gains. [Article]. Psychological Reports, 19(1), 115-\&.

Schulenberg, J. E., \& Maggs, J. L. (2002). A developmental perspective on alcohol use and heavy drinking during adolescence and the transition to young adulthood. Journal of Studies on Alcohol, 54-70.

Straehler-Pohl, H., Fernandez, S., Gellert, U., \& Figueiras, L. (2014). School mathematics registers in a context of low academic expectations. [Article]. Educational Studies in Mathematics, 85(2), 175-199, doi:10.1007/s10649-013-9503-5.

Sweeting, H., \& West, P. (2001). Social class and smoking at age 15: the effect of different definitions of smoking. Addiction, 96(9), 1357-1359, doi:10.1080/09652140120070409.

Turner, J., Perkins, H. W., \& Bauerle, J. (2008). Declining negative consequences related to alcohol misuse among students exposed to a social norms marketing intervention on a college campus. Journal of American College Health, 57(1), 85 - 93.

Van Houtte, M., \& Van Maele, D. (2012). Students' sense of belonging in technical/vocational schools versus academic schools: The mediating role of faculty trust in students. [Article]. Teachers College Record, 114(7), 36.

Webb, E., Ashton, C. H., Kelly, P., \& Kamali, F. (1998). An update on British medical students' lifestyles. Medical Education, 32(3), 325-331.

Wicki, M., Kuntsche, E., \& Gmel, G. (2010). Drinking at European universities? A review of students' alcohol use. [Review]. Addictive Behaviors, 35(11), 913-924, doi:10.1016/j.addbeh.2010.06.015. 